\title{
Wie attribuieren die Schüler die Ursachen des Misserfolgs
}

\section{Svetlana Stančeková und Eva Stranovská}

The article deals with the subject of attribution. Attribution is defined by the way we ascribe causes of our successes and failures. The attribution processes regularly applied by a certain person result in different individual attribution styles. In this article, we are interested in the attribution processes going on in school during second language acquisition. The study examines whether there is a correlation between the attribution of possible failures to certain instances and the overall performance in the German language.

Attribution - attribution style - causes - failure - AASQ

Der Beitrag behandelt das Thema der Attribution. Attribution bedeutet, in welchen Bereichen und auf welche Art und Weise man die Ursachen für seine Erfolge und Misserfolge zuschreibt. Aus dem typischen Attributionsprozess einer Person resultiert ihr Attributionsstil. Uns interessiert dieses Attributionsverfahren in der Schule beim Fremdsprachenerwerb. In der Untersuchung wurde geprüft, ob die Attribution des Misserfolgs mit der Leistung im DaF-Unterricht zusammenhängt.

Attribution - Attributionsstil - Ursachen - Misserfolg - AASQ-Fragebogen

\section{Einleitung}

Nichts geschieht ohne Grund, und für Menschen ist es typisch, Gründe dafür zu suchen, warum die anderen so handeln, wie sie handeln, und was sie dazu führt. Zur Erklärung des Verhaltens des Individuums tragen die Attributionstendenzen bei. Attribution bedeutet so viel wie „Ursachenzuschreibung“.

Die Attribution hängt mit der Suche nach den Gründen des Verhaltens von uns selbst und auch von anderen zusammen. Die Art unseres Verhaltens anderen Menschen gegenüber hängt in hohem Maße davon ab, welche Gründe diesem Verhalten zugeschrieben werden. Die Attributionen sind deswegen sehr wichtig, um die soziale Interaktion mit anderen Menschen effektiv bewältigen zu können.

\section{Attributionstheorien}

Attribution ist ein sozio-psychologischer Begriff. Der Begriff wurde zuerst im Rahmen der sozialen Psychologie entwickelt, es zeigte sich aber, dass die Attributionen ihre Anwendung auch in anderen Bereichen, z. B. in der pädagogischen Psychologie finden. Die Menschen haben seit jeher die Tendenz, dem Geschehen um sich herum, dem eigenen Verhalten oder dem Verhalten der anderen einen Grund zuzuschreiben. Dieses Attributionsverfahren erleichtert ihnen die Orientierung in der Welt (Nakonečný 1998). Dabei interessieren sich die Menschen nicht so 
sehr dafür, was die anderen tun, sondern eher dafür, warum sie dies oder jenes tun. Deswegen nehmen sie Attributionen vor (Gilbert und Malone 1995).

Bei der kausalen Attribution handelt es sich um eine Art, wie subjektiv eine Verbindung zwischen der Ursache und der Folge des Verhaltens hergestellt werden kann.

Systematisch hat den Attributionsprozess der in den USA wirkende österreichische Psychologe Fritz Heider (1958) untersucht. Er kann als Begründer der ersten Attributionstheorie gelten, die auch als "naive Analyse des Verhaltens“ (Heider 1958: 79) bezeichnet wurde. Heider (1958) unterscheidet zwei Gruppen von Attributionen:

- dispositionale Verursachung - die Ursache des Verhaltens liegt in der Person, d. h. sie ist internal,

- situationale Verursachung - die Ursache des Verhaltens liegt in der Situation, nicht in der Person, d. h. sie ist external.

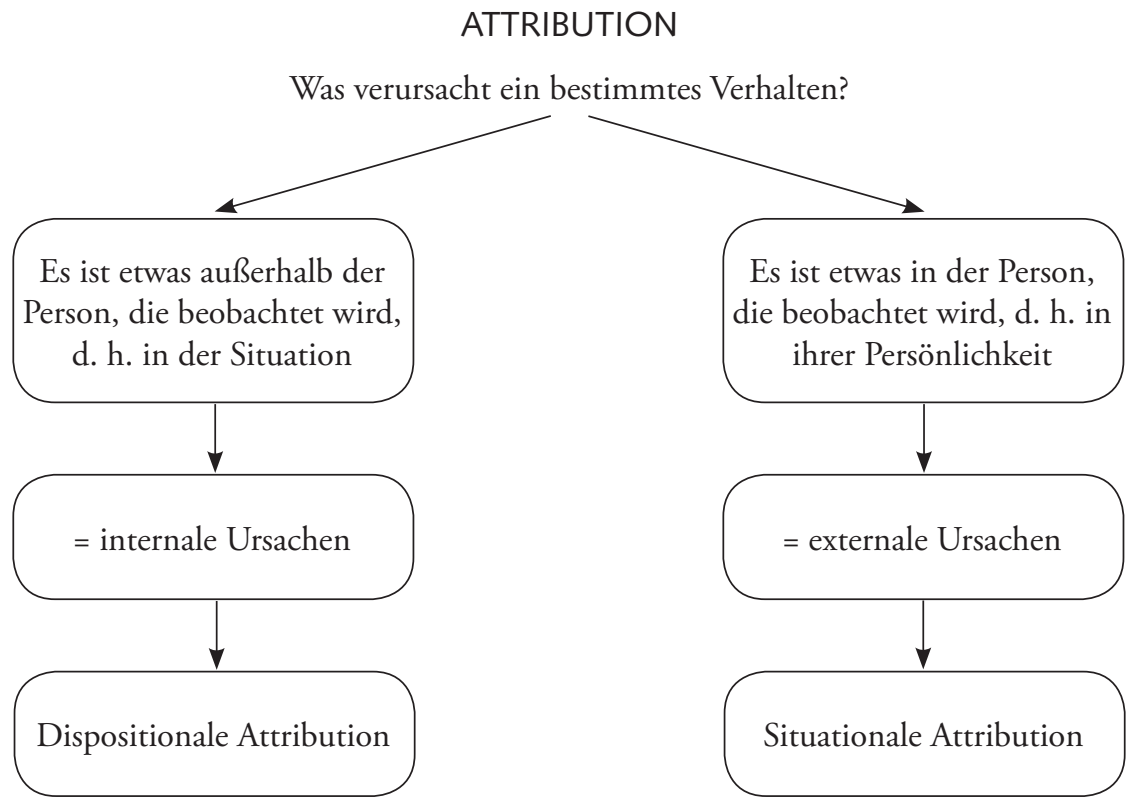

Abb. 1: Dispositionale und situationale Attribution (Quelle: selbst erstellt) 
Das ursprüngliche Konzept der Attribution hat später der amerikanische Psychologe Kelley (1967) weiterentwickelt. Sein Konzept zählt zu den präzisesten Theorien im Bereich der Attribution und wird in zwei Unterkonzepte eingeteilt, je nach der Menge der Information, die der Beobachter zur Verfügung hat:

1. Das Kovariationsprinzip - wenn einem viele Informationen zur Verfügung stehen.

2. Das Modell der kausalen Schemata - wenn man nur über sehr beschränkte Informationen verfügt.

Das Kovariationsprinzip (Kelley 1973) beschreibt die Attribution, die auf mehreren Beobachtungen basiert (z. B. kennt der Beobachter den Akteur und sein Verhalten in verschiedenen Situationen). Neben den Beobachtern und den Akteuren wird auch die Zeit berücksichtigt. Die Auswirkung wird der Ursache zugeschrieben, mit der sie über die Zeit kovariiert.

Die Informationen aus den Beobachtungen sind durch drei Merkmale gekennzeichnet:

- Konsensus,

- Konsistenz,

- Distinktheit.

Der Konsensus beurteilt die Stabilität des Verhaltens des Akteurs im Vergleich mit anderen Menschen, d. h. wie sein Verhalten gewöhnlich ist, im Vergleich mit dem Verhalten von anderen. Die Konsistenz beurteilt die Stabilität des Verhaltens des Akteurs zu unterschiedlichen Zeitpunkten, d. h. ob sein Verhalten in einer bestimmten Situation immer gleich ist. Die Distinktheit bewertet die Stabilität der Akteursreaktion in unterschiedlichen Umgebungen, d. h. ob das Verhalten des Akteurs gleich bleibt, auch wenn sich die Umwelt ändert.

Diese Merkmale können entweder niedrig oder hoch sein. Bei hohem Konsensus, hoher Distinktheit und/oder hoher Konsistenz schreibt der Beobachter das Verhalten der Situation zu. Bei niedrigem Konsensus, niedriger Distinktheit und/ oder hoher Konsistenz schreibt der Beobachter das Verhalten der Persönlichkeit des Akteurs zu.

\begin{tabular}{|l|l|l|l|}
\hline Attribution & Konsensus & Konsistenz & Distinktheit \\
\hline Internal (Persönlichkeit) & niedrig & hoch & niedrig \\
\hline External (Situation) & hoch & hoch & hoch \\
\hline Ausnahme & hoch oder niedrig & niedrig & hoch oder niedrig \\
\hline
\end{tabular}

Tab. 1: Kovariationstabelle

(Quelle: selbst erstellt)

Das Modell der kausalen Schemata beschreibt die Attribution, die auf einer einzelnen Beobachtung basiert. Kausale Schemata oder Konfigurationen sind 
allgemeine Vorstellungen, Überzeugungen, Vorurteile oder Erfahrungen, die dem Beobachter helfen, die Attribution auch dann durchzuführen, wenn er nicht über genug Information verfügt. Meistens ist dies dann der Fall, wenn er den Akteur nicht kennt.

In den achtziger Jahren hat sich auch Weiner (1986) mit der Attribution beschäftigt. Weiner hat die Attribution im Bereich der Leistungsmotivation erweitert. Er hat ein Klassifikationssystem entwickelt, in dem die Personen die Ursachen mit Erfolg oder Misserfolg attribuieren. Im einfachen Fall wird das $2 \times 2$-Schema der Leistungsattribution verwendet (Mandl und Kopp 2008: 180):

\begin{tabular}{|l|l|l|}
\hline & Internal & External \\
\hline Stabil & Fähigkeiten & Aufgabenschwierigkeit \\
\hline Variabel & Anstrengung & Glück \\
\hline
\end{tabular}

Tab. 2: $2 \times 2$-Schema der Leistungsattribution

Nach dem zweidimensionalen Konzept griff Weiner (1986) zum dreidimensionalen Modell der Ursacheneinteilung. Die Ursachen für Erfolg oder Misserfolg lassen sich in drei Dimensionen einteilen:

- Lokation,

- Stabilität,

- Kontrollierbarkeit.

Im Rahmen der Dimension der Lokation können Ursachen von Prüfungserfolg oder -misserfolg external oder internal lokalisiert werden. $\mathrm{Zu}$ den externalen Ursachen gehören die Aufgabenschwierigkeit, der Zufall oder die Laune des Lehrers. $\mathrm{Zu}$ den internalen Ursachen gehören die Fähigkeiten, die Tüchtigkeit oder der Gesundheitszustand des Akteurs.

Die Dimension der Stabilität bewegt sich zwischen den Polen stabil und variabel. Die Ursache kann entweder relativ stabil, d. h. lang andauernd wirksam sein, z. B. Intelligenz, Fähigkeiten oder Aufgabenschwierigkeit, oder sie kann variabel sein, d. h. die Ursache tritt kurzfristig ein, z. B. Zufall oder momentane Stimmungen. Diese Dimension hat einen stark motivierenden Charakter (Hrabal, Man und Pavelková 1989).

Es ist zu erwarten, dass die Berücksichtigung der Dimension „Kontrollierbarkeit“ zu einer besseren Klassifikation der Ursachen für den Erfolg oder Misserfolg bei Prüfungen führt. Sie hängt mit der Bewertung anderer Personen zusammen und gibt Auskunft darüber, ob die Person ihre Handlungen in bestimmter Situation steuern kann oder eher nicht. 
Insgesamt werden die Ursachen in acht Gruppen eingeteilt (Weiner 1986):

\begin{tabular}{|l|l|l|l|l|}
\hline Ursachen & Kontrollierbar & Kontrollierbar & Unkontrollierbar & Unkontrollierbar \\
\hline & Stabil & Variabel & Stabil & Variabel \\
\hline Internal & Wissen & Anstrengung & Fähigkeiten & Laune \\
\hline External & $\begin{array}{l}\text { Hilfe eines } \\
\text { Freundes }\end{array}$ & $\begin{array}{l}\text { Hilfe eines } \\
\text { Fremden }\end{array}$ & $\begin{array}{l}\text { Aufgaben- } \\
\text { schwierigkeit }\end{array}$ & Glück, Zufall \\
\hline
\end{tabular}

Tab. 3: Arten der Ursachen nach Externalität, Stabilität und Kontrollierbarkeit (Quelle: selbst erstellt)

Die Menschen erklären sich ihren Erfolg unterschiedlich, schreiben ihm verschiedene Ursachen zu. Jeder kann auf diese Art und Weise einen eigenen Attributionsstil entwickeln.

\section{Attributionsstile}

Bestimmte Attributionen können typisch für eine bestimmte Person werden. Wenn dies der Fall ist, spricht man von „Attributionsstilen“. Attributionsstile werden als „habituelle Voreinstellungen im Hinblick auf die Zuschreibung von Ursachen für bereits eingetretene Ereignisse" definiert (Weber und Rammsayer 2012: 101).

Jeder von uns hat einen anderen Attributionsstil, wir unterscheiden uns also in der Ursachenzuschreibung und oft haben wir eine relativ stabile und bereits gelernte Tendenz, wo wir die Ursachen des Verhaltens von uns selbst und von den anderen suchen. Im Schulbereich ist es möglich, vier typische Attributionsstile von Schüler/ innen zu beschreiben. Die Hauptdeterminanten sind dabei das Anfangsniveau des Selbstbewusstseins, die Erwartung, die Leistungsbewertung und das Verhalten aus Sicht der anerkannten Kriterien des schulischen Erfolgs (Kusák und Dařílek 2000).

Schüler mit selbstpräsentierendem Attributionsstil nehmen jeden Erfolg an, akzeptieren aber keine Misserfolge. Sie schreiben den Erfolg vor allem dispositionalen Ursachen (z. B. Fähigkeiten) zu.

Ein selbstprotektiver Attributionsstil kommt im Falle eines unerwarteten Misserfolgs vor. Der/die Schüler/in lehnt die eigene Verantwortung für sein Versagen $\mathrm{ab}$ und schreibt den Misserfolg vor allem situationalen Ursachen zu, z. B. Pech, Aufgabenschwierigkeit, subjektive Bewertung des Lehrers u. a.

Bei einem kontraselbstpräsentierenden Attributionsstil wird die Verantwortung für den Misserfolg unter Hinweis auf situationale Ursachen (Glück, Zufall) abgelehnt. Einen solchen Stil bevorzugen vor allem Schüler/innen mit niedrigem Selbstbewusstsein.

Ein kontraselbstprotektiver Attributionsstil tritt auf, wenn der/die Schüler/in den Misserfolg dispositionalen Ursachen (Mühe, Fähigkeiten, Fleiß) zuschreibt. Der/die Schüler/in ist bereit, für das Versagen die volle Verantwortung zu übernehmen. 
Er/sie braucht keinen speziellen Schutzmechanismus, um in solchen Situationen die eigenen Persönlichkeitsqualitäten zu schützen.

\section{Untersuchungsziel und Untersuchungsfrage}

Die Attributionstheorie wird v. a. im Zusammenhang mit der Motivation beim Lernen (Mandl und Kopp 2008, Plevová 2007, Weiner 1979) untersucht. Auch wir halten die Untersuchung der Attributionsstile in Verbindung mit Erfolgen und Misserfolgen beim Fremdsprachlernen für wesentlich: Jedes Individuum denkt nämlich über seine Erfolge und Misserfolge beim Fremdsprachenerwerb nach und sucht nach Möglichkeiten, wie es erfolgreich sein kann. Aus diesen Überlegungen hat sich das Untersuchungsziel ergeben, herauszufinden, wie die Attribution des Erfolgs oder Misserfolgs mit den Leistungen im DaF-Unterricht korreliert. Für die Untersuchung wurde folgende Untersuchungsfrage formuliert:

Wie hängen die Attributionen des Misserfolgs mit der Leistung in der Fremdsprache Deutsch zusammen?

\section{Untersuchungsmethoden und Probanden}

Die Untersuchung wurde in den Jahren 2016 und 2017 durchgeführt. 135 Schüler der Grundschule und des Gymnasiums in Bratislava in der Slowakischen Republik haben daran teilgenommen. Das Durchschnittsalter war 15 Jahre. Deutsch ist für die Probanden die zweite Fremdsprache. Die Schüler haben den JASQ-Fragebogen (Junior Attributional Style Questionnaire, adaptiert nach Peterson und Barrett 1987) und einen didaktischen Test erhalten und ausgefüllt.

Der JASQ-Fragebogen geht von dem AASQ-Fragebogen (Academic Attributional Style Questionnaire) aus. Dieser wurde von Peterson und Barrett (1987) entwickelt und dient zur Untersuchung von Optimismus und Pessimismus als unterschiedlichen Attributionsstilen. Der AASQ-Fragebogen ist auf Student/innen und ihre Lebenserfahrungen abgestimmt. Er enthält negative hypothetische Situationen, mit denen der Student/die Studentin in Kontakt kommen kann. Der JASQ-Fragebogen wurde an die Situation von Schülern an der Grund- und Mittelschule angepasst. Im Fragebogen gibt es zwölf Situationen, wie etwa Misserfolg beim Test, die Drohung der Jahrgangswiederholung oder die Unmöglichkeit, ein Buch für den Unterricht zu beschaffen. Jede von diesen Situationen enthält vier Fragen. Die erste fragt nach der Hauptursache, nach dem Hauptgrund dieses negativen Ereignisses. Der Proband soll sie aufschreiben. Die anderen drei Fragen führen die Ursachenforschung weiter. Der Proband soll dabei auf einer siebenstufigen Likertskala markieren, inwieweit er diese Ursache beeinflussen konnte, ob sie sich auch in Zukunft wiederholen kann und ob diese Ursache nur Situationen der beschriebenen Art oder auch andere Situationen beeinflussen kann. 
Ein didaktischer Test ergänzt den JASQ-Fragebogen und misst die Leistung der Probanden in DaF. Es handelt sich dabei um Tests auf den Niveaustufen A2 und B1 des Gemeinsamen europäischen Referenzrahmens.

\section{Ergebnisse}

In Tabelle 4 ist die deskriptive Statistik ausgewählter Situationen aus dem JASQFragebogen dargestellt. Jede Situation enthält vier Fragen (a, b, c, d), wobei Frage a nicht ausgewertet wird. Es handelt sich um einen standardisierten Fragebogen nach Peterson und Barrett (1987), in dem die Antwort a für den Probanden hilfreich ist, um weitere drei Fragen zu beantworten. Deswegen wird sie standardmäßig nicht ausgewertet. Die Fragen b, c, d repräsentieren die einzelnen Dimensionen der Ursachen. In Frage b bestimmt der Proband, ob er die Ursache beeinflussen konnte oder nicht - das ist die Dimension der Internalität. In Frage c bezeichnet der Proband, ob die Ursache auch in Zukunft vorkommen könnte - das ist die Dimension der Stabilität. Frage d untersucht, ob die Ursache nach Ansicht des Probanden nur im beschriebenen Situationstyp gilt oder ob auch andere Situationen in anderen Bereichen seines Lebens dieselbe Auswirkung hätten - das ist die Dimension der Globalität.

Auf Grund dieser drei Dimensionen definierte Seligman (1998) den optimistischen und pessimistischen Explanationsstil. Optimisten schreiben negativen Situationen externale, variable und spezifische Ursachen zu. Dagegen bezeichnen Pessimisten diese Ursachen als internal, stabil und global (Peterson und Barrett 1987). Je höher die Punktzahl ist, die der Proband erreicht, desto pessimistischer ist sein Attributionsstil und umgekehrt.

\begin{tabular}{|l|l|l|l|l|l|l|}
\hline Situation & Frage & Mean & Median & Min & Max & Stdev \\
\hline \multirow{4}{*}{2} & b & 5.039216 & 5 & 1 & 7 & 1.768378 \\
\cline { 2 - 7 } & c & 4.666667 & 5 & 1 & 7 & 1.543644 \\
\cline { 2 - 7 } & d & 3.823529 & 4 & 1 & 7 & 1.742945 \\
\hline \multirow{4}{*}{4} & b & 5.386139 & 6 & 1 & 7 & 2.009827 \\
\hline & c & 3.742574 & 4 & 1 & 7 & 2.152457 \\
\hline \multirow{4}{*}{6} & d & 3.811881 & 4 & 1 & 7 & 2.086686 \\
\hline \multirow{2}{*}{} & b & 3.10101 & 3 & 1 & 7 & 1.950902 \\
\cline { 2 - 7 } & c & 4.363636 & 4 & 1 & 7 & 1.514923 \\
\cline { 2 - 7 } & d & 3.474747 & 4 & 1 & 7 & 1.66196 \\
\hline
\end{tabular}




\begin{tabular}{|l|l|l|l|l|l|l|}
\hline \multirow{4}{*}{7} & b & 5.833333 & 7 & 1 & 7 & 1.659556 \\
\cline { 2 - 8 } & c & 3.794118 & 4 & 1 & 7 & 1.900173 \\
\cline { 2 - 8 } & d & 4.215686 & 4 & 1 & 7 & 2.022778 \\
\hline \multirow{4}{*}{9} & b & 2.950495 & 2 & 1 & 7 & 2.165069 \\
\cline { 2 - 8 } & c & 4.19802 & 4 & 1 & 7 & 1.691271 \\
\cline { 2 - 8 } & d & 3.267327 & 3 & 1 & 7 & 1.994448 \\
\hline
\end{tabular}

Tab. 4: Deskriptive Statistik ausgewählter Situationen Legende: MEAN: Durchschnittswert, STDEV: Standardabweichung, MIN: Minimalwert, MAX: Maximalwert.

Frage 2: Sie bekommen schlechte Note im Test oder in der mündlichen Prüfung. Mit dem Durchschnitt 5 hat die deskriptive Statistik gezeigt, dass die Probanden in dieser Situation eher interne und stabile Ursachen für ihren Misserfolg suchen. Die Dimension der Globalität neigt leicht zu den spezifischen Ursachen. Die Probanden sind im Durchschnitt eher pessimistisch.

Frage 4: Ihnen droht das Durchfallen in der Schule und damit das Wiederholen des Schuljahrs.

Die Dimension der Internalität weist stark auf die internen Ursachen, in den anderen Dimensionen sehen die Probanden die Ursachen eher als variabel und spezifisch. Bei dieser Frage weisen die Probanden im Durchschnitt eher Pessimismus auf.

Frage 6: Sie können eine einzige Aufgabe von allen zwanzig nicht lösen.

Die Probanden bezeichneten die Ursachen als extern und spezifisch. Die Werte in der Dimension "Stabilität" bewegten sich somit im mittleren Bereich, sie wiesen weder ausgeprägte Abweichungen in Richtung ,variabel“ noch in Richtung „stabil“ auf. Insgesamt neigten die Probanden eher zu Optimismus als Attributionsstil.

Frage 7: Sie müssen die Schule wegen nicht ausreichender schulischer Leistung verlassen.

In der Dimension „Internalität“ wird die Ursache von den Probanden eindeutig internal gesehen. Allerdings halten die Probanden die Ursachen in den anderen Dimensionen für variabel und eher global. Sie sind eher pessimistisch.

Frage 9: Es gelingt Ihnen nicht, ein Buch in der Bibliothek zu finden.

Hier sehen die Probanden die Ursachen für die negative Situation eher in dem externen und spezifischen Bereich, die Dimension der Stabilität bleibt dabei durchschnittlich. Die Probanden sind eher Optimisten. 


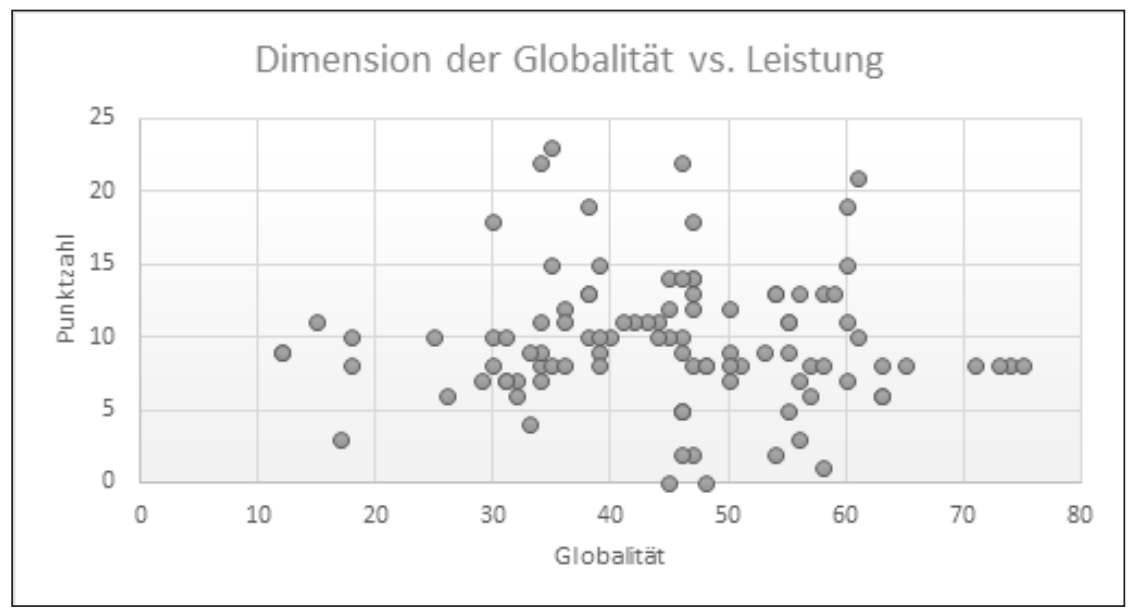

Abb. 2: Die Punktzahl aus dem didaktischen Test im Zusammenhang mit der Dimension der Globalität

In Abbildung 2 ist der Zusammenhang der Leistung, d. h. der in einem didaktischen Test erzielten Punktzahl mit der Punktzahl aus dem JASQ-Fragebogen in der Dimension der Globalität zu sehen. Die Probanden mit höherer Leistung, d. h. diejenigen, die mehr als 15 Punkte erreicht haben, halten auch die Ursachen für eher global. Das bedeutet, dass die Ursachen nach Ansicht der Probanden allgemein sind und auch in anderen Situationen auftreten; sie sind nicht nur für diesen konkreten Situationstyp kennzeichnend. Diese Probanden sind eher pessimistisch; Misserfolge nehmen sie in allen Bereichen als bedeutsam wahr. Die anderen Probanden mit weniger Punkten im Test weisen keinen spezifischen Attributionsstil im Zusammenhang mit der Leistung auf; sie halten die Ursachen für die negative Situation, der sie nach der Beschreibung in der Erhebung ausgesetzt sind, sowohl für spezifisch als auch für global.

\section{Diskussion}

Unser Schulsystem ist so eingestellt, dass sich die Schüler und Schülerinnen wenigstens teilweise auf Leistung hin orientieren. Es werden Ziele festgelegt, die sie erreichen sollten. Wie ihnen das gelingt, empfinden sie dann als Erfolg oder Misserfolg. Damit hängt die Leistungsmotivation zusammen. Es gibt nicht viele Untersuchungen, die sich mit der Attribution des Erfolgs oder Misserfolgs im Zusammenhang mit der Leistung in der Fremdsprache beschäftigen. Die Attributionsprozesse von erfolgreichen und erfolglosen Schülern in unterschiedlichen Fächern haben Dařilek und Kaločová (1991) untersucht. Es zeigte sich, dass erfolgreiche Schüler ihren 
Erfolg eher stabilen und spezifischen Ursachen zugeschrieben haben. Umgekehrt sahen weniger erfolgreiche Schüler die Ursachen für ihren Misserfolg im Zufall und in externalen Ursachen, wie etwa der Schwierigkeit des Lernstoffes.

In der Untersuchung hatten wir vor festzustellen, ob und wie der Misserfolg und die Wahrnehmung der negativen Situationen mit der Leistung zusammenhängen. Die Ergebnisse zeigen keine so starke Korrelation wie bei Dařílek und Kaločová (1991): Die Leistungen in DaF und die Attribution des Misserfolgs sind relativ unabhängig voneinander. In der Gruppe der Probanden mit höherer Leistung ist allerdings beim Misserfolg eine Tendenz zum Pessimismus zu beobachten. Sie nehmen den Misserfolg offensichtlich ernst und denken trotz überdurchschnittlicher Leistungen, dass negative Erfahrungen in allen Bereichen ihres Lebens wirken. Das stellt ihren Explanationsstil dar, das bedeutet, ihre Einstellung zur Welt, ihre Art der Weltsicht (Křivohlavý 2012).

Bei allen anderen Probanden ist es nicht eindeutig, ob die Leistung und erreichten Ziele die Attribution beeinflussen. Darauf wirken nämlich mehrere Faktoren, nicht nur die Wahrnehmung von Erfolg und Misserfolg. Pavelková (2002) beschreibt die Schule als eine Umgebung, in der die Schüler sowohl Erfolge als auch Misserfolge erleben. Zur Erfolglosigkeit eines Schülers führt eine Kombination von mehreren Faktoren, wobei es sich zum einen um die Persönlichkeit des Schülers handelt, zum anderen aber auch um die Persönlichkeit der Lehrer, mit denen der Schüler konfrontiert ist (Stančeková 2017). Der Explanationsstil ist damit nur einer von vielen Faktoren, die das Erleben von Misserfolg bestimmen.

\section{Zusammenfassung}

Die Leistung in der Fremdsprache ist eine komplexe Erscheinung, die von vielen Faktoren beeinflusst wird. Einen dieser Faktoren stellt die Attribution des Erfolgs und Misserfolgs dar. Unsere Untersuchung hat zwar nur einen geringen Zusammenhang zwischen dem Erfolg und dem Attributionsstil gezeigt, eröffnet damit allerdings auch neue Wege zu weiteren Untersuchungen. Die Attribution des Erfolgs und Misserfolgs könnte z. B. mit dem Interesse an der Fremdsprache korreliert, auf anderen Niveaustufen (von A1 bis C2) untersucht oder in die einzelnen Komponenten des Attributionsverfahrens aufgelöst werden. Interessant könnten auch die Analyse und der Vergleich des optimistischen und pessimistischen Explanationsstils beim Fremdsprachenerwerb sein. 


\section{Literaturverzeichnis}

Dařílek, Pavel / Kaločová, Irena (1991): Přisuzování př́ǐcin úspěšných a neúspěšných žáků. In: Psychologové studentưm. Praha, SPN. S. 25-30.

Gilbert, Daniel T. / Malone, Patrick S. (1995): The correspondence bias. In: Psychological Bulletin 117/1/1995. S. 21-38.

Heider, Fritz (1958): The Psychology of Interpersonal Relations. New York, Wiley.

Hrabal, Vladimír / Man, František / Pavelková, Isabella (1989): Psychologické otázky motivace ve škole. Praha, SPN.

Kelley, Harold. H. (1967): Attribution Theory in Social Psychology. In: Nebraska Symposium on Motivation. Lincoln, University of Nebraska Press. S. 192-238.

Kelley, Harold H. (1973): Process of Causal Attribution. In: American Psychologist 28/2/1973. S. 107-128.

Křivohlavý, Jaro (2012): Optimismus, pesimismus a prevence deprese. Praha, Grada Publishing. Kusák, Pavel / Dař́lek, Pavel (2000): Pedagogická psychologie. Olomouc, UPOL.

Mandl, Heinz / Kopp, Birgitta (2008): Ursachenzuschreibungen aus Sicht der Attributionstheorie. In: Zumbach, Jörg / Mandl, Heinz (Hg.): Pädagogische Psychologie in Theorie und Praxis. Göttingen, Hogrefe. S. 177-183.

Nakonečný, Milan (1998): Encyklopedie obecné psychologie. Praha, Academia.

Pavelková, Isabella (2002): Motivace žákủ k učeni. Perspektivni orientace žákủ a časový faktor $v$ žákovské motivaci. Praha, PedF UK.

Peterson, Christopher / Barrett, Lisa (1987): Explanatory style and academic performance among university freshman. In: Journal of Personality and Social Psychology 53/3/1987. S. 603-307.

Plevová, I.: Kauzálni atribuce aneb jak pátráme po přičinách životnich událostí. Olomouc: Hanex, 2007.

Seligman, Martin (1998): Learned Optimism. New York, Pocket Books.

Stančeková, Svetlana (2017): Endogene Faktoren im Fremdsprachenerwerb. In: Motus in verbo 6/1/2017. S. 39-52.

Weber, Hannelore / Rammsayer Thomas (2012): Differentielle Psychologie Persönlichkeitsforschung. Göttingen, Hogrefe.

Weiner, B. (1979). A theory of motivation for some classroom experiences. In: Journal of Educational Psychology. 71. S. 3-25.

Weiner, Bernard (1985): An attributional theory of achievement motivation and emotion. In: Psychological Review 92/1985. S. 548-573.

Weiner, Bernard (1986): An Attributional Theory of Motivation and Emotion. New York, Springer Verlag.

Diese Arbeit wurde von der Agentur für die Unterstützung der Forschung und Entwicklung auf Grund des Vertrags Nr. APVV-14-0446 und APVV-17-0071 gefördert.

Svetlana Stančeková

Philosoph-Konstantin-Universität in Nitra

svetlana.stancekova@ukf.sk
Eva Stranovská

Philosoph-Konstantin-Universität in Nitra

estranovska@ukf.sk 